\title{
Number processing in temporal lobe epilepsy
}

\author{
M Delazer, A Gasperi, L Bartha, E Trinka, T Benke
}

J Neurol Neurosurg Psychiatry 2004;75:901-903. doi: 10.1136/jnnp.2003.123614

\begin{abstract}
Background: Specific cognitive impairments have been found in association with mesial temporal lobe epilepsy (TLE), such as deficits in declarative memory or verbal abilities. No attention has been paid so far to possible deficits in number processing.

Objective: To investigate deficits in number processing in patients with TLE.

Methods: Numerical abilities were assessed in 28 right handed patients with medically intractable unilateral TLE and in a control group.

Results: No differences between patients and controls were found in analogue number processing with Arabic input, in a comparison task, or in simple addition and simple subtraction; however, there were significant group differences in tasks with verbal input, in simple division, in complex mental calculation, in a semantic knowledge task, and in conceptual tasks. Only minor differences were found between patients with right and left TLE.

Conclusions: While numerical deficits may be expected in patients with left sided TLE, it is open for discussion why patients with right sided TLE also show numerical deficits.
\end{abstract}

$\mathrm{S}$ pecific cognitive impairments have been found to be associated with mesial temporal lobe epilepsy (TLE), such as deficits in declarative memory, ${ }^{1}$ face/name processing, ${ }^{2}$ or language functions. ${ }^{3}$ However, little attention has been paid so far to possible deficits in number processing. Impairment of number processing is common with focal and diffuse brain disease and is typically associated with left posterior lesions. ${ }^{4-6}$ Though deficits in number processing and calculation are severe handicaps in everyday life, acquired numerical deficits are often not appropriately diagnosed. While assessment of number processing is mostly limited to single subtests of larger batteries, a more specific diagnosis oriented at current neuropsychological models is needed. A recent neuropsychological model ${ }^{78}$ proposes three numerical systems: first, operations based on the processing of quantities would be supported by both hemispheres-in particular by the horizontal part of the bilateral intraparietal sulcus. Second, verbal aspects of number processing (for example, reciting the multiplication tables) would be mediated by language relevant areas of the left hemisphere and the left angular gyrus. Third, the visual Arabic system would involve the inferior temporo-occipital cortex and the posterior superior parietal lobes. It should be noted, however, that this model is still tentative in some aspects and is debated in various studies. ${ }^{9}$

In the present study number processing was assessed in two groups of patients with unilateral mesial TLE (right or left). Following the triple code model it was predicted that both groups should perform well in tasks of quantity based processing, which are thought to be bilaterally represented (comparison, analogue magnitude processing with Arabic input, simple subtraction and addition). On the other hand, patients should show more difficulty with skills that are thought to be more lateralised (multiplication, division, complex mental calculation, tasks with verbal input, semantic number knowledge). This should be particularly true for patients with left hemisphere dysfunction. ${ }^{10}{ }^{11}$ We further expected deficits in conceptual knowledge of arithmetic in both patients groups. Deficits in conceptual knowledge have been observed after left parietal lesions affecting number processing. ${ }^{12}$ However, it was hypothesised that such a complex ability-which involves drawing inferences and manipulating numerical information-relies on the interplay of different brain regions and may thus be affected in both TLE groups. ${ }^{13}$

\section{METHODS}

\section{Participants}

Patients were recruited during a presurgical evaluation programme which included detailed clinical and neurological examination. All patients underwent high resolution magnetic resonance imaging (MRI) and intensive video-EEG monitoring with scalp and sphenoidal electrodes for an average of five days. Interictal spikes were quantified according to frequency and location. Ictal EEG was classified according to localisation, morphology, and spatio-temporal evolution. All patients underwent a comprehensive neuropsychological examination and Wada test (all were left hemispheric language dominant). Interictal and ictal SPECT studies were done in patients with ambiguous results on non-invasive video-EEG monitoring. All patients had medically intractable unilateral temporal lobe epilepsy. Seizure focus localisation was based on concordant findings in the presurgical evaluation. Fifteen patients with left TLE and 13 patients with right TLE-comparable for age, education, duration of disease, and general intelligence-were included in the study, along with 55 controls for the numerical tasks, matched for age and education.

\section{Numerical tasks}

- Analogue number scale $(\mathrm{n}=12)$. Subjects have to choose (among three alternatives) the position corresponding to a given Arabic numeral (or number word) on an analogue number scale. In half the trials the number scale extends from 0 to 100, in the other half from 0 to 50. Half the items were presented with Arabic numerals, half with number words.

- Arithmetic facts (eight problems for each operation; $\mathrm{n}=32$ ). All problems are presented in Arabic format (for example, $3 \times 4)$ and are answered verbally ("twelve"). Problems are blocked by operation.

- Mental calculation (five problems each for addition, subtraction, and multiplication; $\mathrm{n}=15$ ). Subjects have to compute mentally the answer to visually presented twodigit problems and to answer verbally.

- Arithmetic principles tasks (15 problems for addition and multiplication; $\mathrm{n}=30$ ). Two related multidigit operations-one with the correct answer, the other with a 


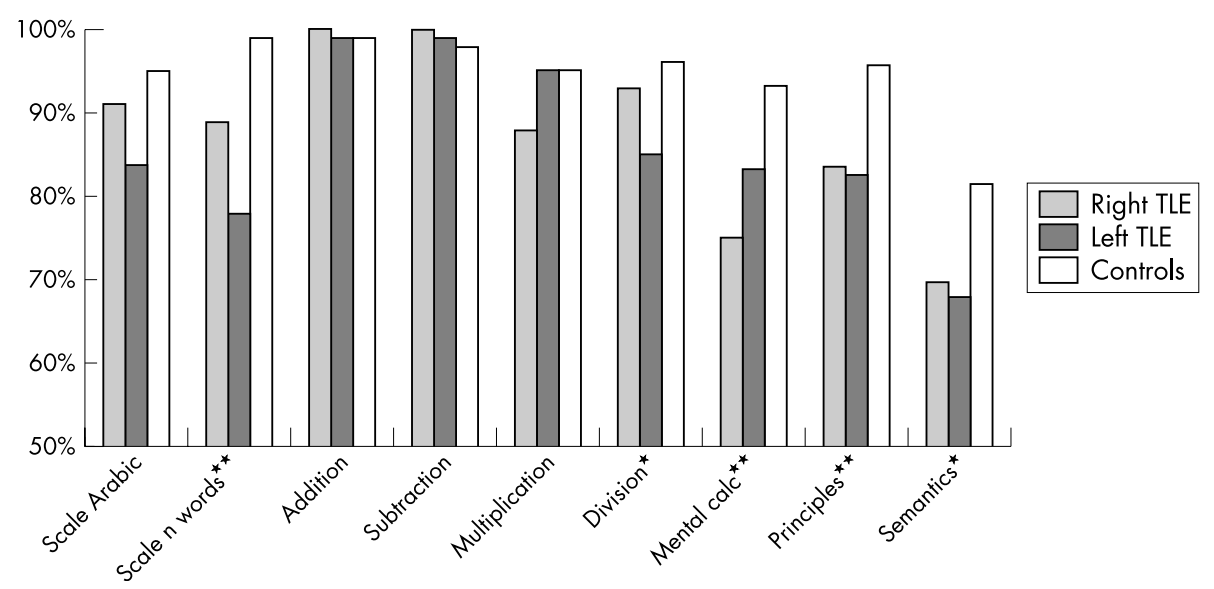

Figure 1 Accuracy rates in numerical tasks. Performance of the three groups in numerical tasks. Significant group differences (Kruskal-Wallis) are indicated by ${ }^{* *}(p<0.0001)$ or ${ }^{*}(p<0.05)$.

blank at the place of the result-are presented to the subject. Subjects are required to give the solution to the second problem without calculating it, but with reference to the first problem.

- Number comparison $(\mathrm{n}=120$ ). Single digits ( 1 to 4,6 to 9) are presented on the computer screen; subjects have to indicate as fast as possible whether the number is smaller or larger than 5 (right button=larger, left button $=$ smaller).

- Semantic number knowledge $(\mathrm{n}=20$; adapted from Crutch and Warrington ${ }^{14}$ ). Subjects have to answer semantic numerical questions such as "How many days are there in a year?". The answer is chosen among four alternatives.

\section{RESULTS}

Comparison of demographic variables showed no differences between groups (Kruskal-Wallis test). The characteristics of the study subjects were as follows (values given as mean $(\mathrm{SD}))$ :

- Left TLE: $\mathrm{n}=15$; age 41.8 (10.6) years; education 11.1 (3.0) years; age at onset 23.0 (17.2) years; duration of disease 18.3 (14.2) years.
- Right TLE: $\mathrm{n}=13$; age 33.5 (13.6); education 10.9 (2.4) years; age at onset 15.1 (14.1) years; duration of disease 18.3 (14.8) years.

- Controls: $\mathrm{n}=55$; age 34.4 (8.2) years; education 10.9 (3.2) years.

Both patient groups scored in the average range for verbal memory, visual learning, object naming, visual scanning, and cognitive flexibility. In the recognition of faces, the right TLE group scored in the low average range, and the left TLE group in the average range. For both groups, reaction times were in the low average range in an alertness task (tonic alertness, phasic alertness), and anxiety and depression scores were average in both groups. Finally, a short form of the Wechsler scale showed an average IQ in both groups (right TLE group, 102.5 (15.0); left TLE group, 104.7 (14.9)). Comparisons using Mann-Whitney tests showed no significant differences between the groups.

\section{Numerical tasks}

Kruskal-Wallis tests (fig 1, table 1) showed significant differences between groups in the verbal number scale task $(\mathrm{p}<0.0001)$, simple division $(\mathrm{p}=0.023)$, the mental calculation task (accuracy, $\mathrm{p}<0.0001$; response times, $\mathrm{p}=0.018$ ),

Table 1 Performance of the three groups in numerical tasks and between group comparisons

\begin{tabular}{|c|c|c|c|c|c|c|c|c|}
\hline & \multirow[b]{2}{*}{$\mathbf{N}$} & \multicolumn{2}{|c|}{ Right TLE } & \multicolumn{2}{|l|}{ Left TLE } & \multicolumn{2}{|l|}{ Control } & \multirow[b]{2}{*}{ Kruskal-Wallis tes } \\
\hline & & Mean & SD & Mean & SD & Mean & SD & \\
\hline Number scale, sum & 12 & 10.77 & 1.74 & 9.73 & 2.02 & 11.64 & 0.59 & $<0.0001$ \\
\hline Input Arabic numerals & 6 & 5.46 & 1.20 & 5.07 & 1.10 & 5.71 & 0.50 & NS \\
\hline Input number words & 6 & 5.31 & 0.75 & 4.67 & 1.18 & 5.93 & 0.26 & $<0.0001$ \\
\hline Arithmetic facts, sum & 32 & 30.54 & 2.50 & 30.33 & 2.69 & 31.71 & 3.47 & \\
\hline Addition & 8 & 8 & 0 & 7.93 & 0.26 & 7.98 & 0.13 & NS \\
\hline Subtraction & 8 & 8 & 0 & 7.93 & 0.26 & 7.91 & 0.35 & NS \\
\hline Multiplication & 8 & 7.08 & 1.50 & 7.60 & 0.63 & 7.65 & 0.62 & NS \\
\hline Division & 8 & 7.46 & 1.20 & 6.87 & 2.10 & 7.73 & 0.78 & 0.023 \\
\hline Mental calculation & 15 & 11.85 & 2.97 & 12.00 & 3.07 & 14.00 & 1.05 & $<0.0001$ \\
\hline RT, mental calculation (s) & & 117.31 & 56.69 & 140.33 & 61.45 & 98.53 & 32.35 & 0.018 \\
\hline Arithmetic principles & 30 & 25.77 & 3.65 & 24.07 & 4.54 & 28.71 & 1.34 & $<0.0001$ \\
\hline Semantic knowledge & 20 & 14.38 & 2.53 & 13.20 & 3.23 & 16.31 & 2.18 & 0.018 \\
\hline \multicolumn{9}{|l|}{ Number comparison } \\
\hline Split 1 RT (ms) & & 631.62 & 120.7 & 653.75 & 138.95 & 512.34 & 56.59 & \\
\hline Split 2 RT (ms) & & 620.37 & 111.46 & 631.14 & 121.98 & 488.73 & 62.29 & \\
\hline Split 3 RT (ms) & & 597.58 & 116.00 & 589.14 & 111.45 & 470.53 & 45.05 & \\
\hline
\end{tabular}


the arithmetic principles task $(\mathrm{p}<0.0001)$, and the semantic knowledge task $(p=0.018)$. Mann-Whitney tests between right and left TLE showed no significant differences, while both groups differed from controls (corrected level of significance, $\mathrm{p}<0.008$; right TLE $v$ controls: verbal number scale task, $\mathrm{p}<0.0001$, mental calculation, $\mathrm{p}=0.0006$, principles task, $\mathrm{p}=0.0008$; left TLE $v$ controls: verbal number scale task, $\mathrm{p}<0.0001$, division, $\mathrm{p}=0.006$; mental calculation, $\mathrm{p}<0.0001$, principles task, $\mathrm{p}<0.0001$, semantic knowledge, $\mathrm{p}<0.0008$ ).

In the number comparison task, mean correct reaction times were assessed by analysis of variance with groups (right TLE, left TLE, controls (subgroup of 13)) as the between-subjects variable, and split $(1,2,3)$ as the withinsubjects variable. The effect of split was highly significant $(\mathrm{F}(2,36)=35.85 ; \mathrm{p}<0.0001)$, as was the effect of group $(\mathrm{F}(2,36)=7.34 ; \mathrm{p}=0.002)$, controls answering faster than patients. No interaction between group and split was found. All groups showed a standard distance effect, with reaction times inversely related to the numerical distance. All groups showed low error rates in the number comparison task (right TLE $0.53 \%$, left TLE $0.65 \%$, controls $0.39 \%$ ).

\section{DISCUSSION}

There were several differences in numerical tasks between the TLE groups and the control group. It was predicted that tasks based on the processing of quantities should not show group differences but that tasks more specifically supported by one hemisphere should show group differences (between TLE groups and controls, but also between the TLE groups). We also assumed that semantic and conceptual knowledge would differ between TLE groups and controls. In fact, the number scale task with Arabic input, simple addition, and simple subtraction did not differ between groups. Moreover, all three groups showed standard distance effects in a number comparison task, ${ }^{8}$ answering significantly faster when the numerical distance was larger than when the distance was smaller. Thus the results confirm the hypothesis that quantity based abilities are not, or are only slightly, affected in TLE patients. Alternatively one could assume that a bilateral hemispheric representation offers better compensation opportunities when there is unilateral dysfunction.

Almost all the other tasks showed significant group differences. The number scale task with verbal input, the simple division task, the mental calculation task (accuracy and answer times), and the semantic knowledge task all differed between groups. These findings are in line with a more lateralised and thus more vulnerable cognitive elaboration. However, it has to be stressed that only minor differences were found between the right and left TLE groups (though the left TLE group had lower scores). While the left TLE group differed from controls in division and in semantic number knowledge, right TLE patients did not. Though the results suggest a small left TLE disadvantage, if any, it emerges from the study that both, right and left TLE patients have difficulties in numerical tasks. While such deficits may have been expected for patients with left TLE, it is open for discussion why patients with right TLE show numerical deficits. There are several possibilities. First, spreading seizures may affect various anatomical areas, including those relevant to numerical processing. ${ }^{15}$ Though numerical processes are predominantly supported by parietal and frontal areas, a recent case study of a calculation prodigy also showed involvement of the medial temporal areas. ${ }^{16}$ Second, multiple regions of the brain-including those critical for numerical processing - may be subject to neuronal cell loss in patients with both right and left TLE. ${ }^{17}$ In fact, it has repeatedly been shown that diffuse brain lesion may lead to generic reduction in processing resources and to marked numerical deficits. ${ }^{18}$ Third, chronic TLE may shift the anatomical representation of cognitive functions. Thus cognitive models developed using data from patients affected by focal brain lesions and neuroimaging data from healthy adults may not apply to patients with TLE.

\section{Authors' affiliations}

M Delazer, A Gasperi, L Bartha, E Trinka, T Benke, Universitätsklinik für Neurologie, Innsbruck, Austria

Competing interests: none declared

Correspondence to: Dr Margarete Delazer, Universitätsklinik für Neurologie, Anichstr 35, 6020 Innsbruck, Austria; margarete.delazer@ vibk.ac.at

Received 16 July 2003

In revised form 3 September 2003

Accepted 9 September 2003

\section{REFERENCES}

1 Helmstaedter C. Effects of chronic epilepsy on declarative memory systems. Prog Brain Res 2002;135:439-53.

2 Hermann BP, Seidenberg M, Haltiner A, et al. Adequacy of language function and verbal memory performance in unilateral temporal lobe epilepsy. Cortex 1992;28:423-33.

3 Glosser G, Salvucci AE, Chiaravalloti ND. Naming and recognizing famous faces in temporal lobe epilepsy. Neurology 2003;61:81-6.

4 Grafman G, Passafiume D, Faglioni $P$, et al. Calculation disturbances in adults with focal hemisphere damage. Cortex 1982;25:37-50.

5 Hecaen $\mathbf{H}$, Angelergues R, Houillier $\mathrm{S}$. Les variétés cliniques des acalculies au cours lésions retrorolandiques: aproche statistique du problème. Rev Neurol $1961 ; 105: 85-103$.

6 Kahn H, Whitaker H. Acalculia: an historical review of localization. Brain Cognition 1991;17:102-15.

7 Dehaene S, Cohen L. Towards an anatomical and functional model of number processing. Math Cogn 1995;1:83-120.

8 Dehaene S, Piazza M, Pinel $P$, et al. Three parietal circuits for number processing. Cogn Neuropsychol 2003;20:487-506.

9 Zago L, Pesenti M, Mellet E, et al. Neural correlates of simple and complex mental calculation. Neuroimage 2001;13:314-27.

10 Jackson M, Warrington EK. Arithmetic skills in patients with unilateral cerebral lesions. Cortex 1986;22:611-20.

11 Butterworth B. The mathematical brain. London: Macmillan, 1999.

12 Delazer M, Benke T. Arithmetic facts without meaning. Cortex 1997;33:697-710.

13 Delazer $M$. Neuropsychological findings on conceptual knowledge of arithmetic. In: Baroody AJ, Dowker A, eds. The development of arithmetic concepts and skills. Mahwah, NJ: Lawrence Earlbaum Associates, 2003:385-408.

14 Crutch S, Warrington E. Acalculia: deficits of operational and quantity number knowledge. J Int Neuropsychol Soc 2001;7:825-34.

15 Klingler J, Gloor P. The connections of the amygdala and of the anterior temporal cortex in the human brain. J Compr Neuroanat 1960;1 15:333-69.

16 Pesenti M, Zago L, Crivello F, et al. Mental calculation in a prodigy is sustained by right prefrontal and medial temporal areas. Nat Neurosci 2001:4:103-7.

17 Liu RS, Lemieux L, Bell GS, et al. Progressive neocortical damage in epilepsy. Ann Neurol 2003;53:312-24.

18 Martini L, Benke T, Domahs F, et al. Everyday numerical abilities in Alzheimer's disease. J Int Neuropsychol Soc 2003;9:871-8. 